\title{
Overexpression of PgSQS1 Increases Ginsenoside Production and Negatively Affects Ginseng Growth Rate in Panax ginseng
}

\author{
Ju-Sun Shim, Ok Ran Lee, Yu-Jin Kim, Jung-Hye Lee, Ju-Han Kim, Dae-Young Jung, Jun-Gyo In, \\ Beom-Soo Lee, and Deok-Chun Yang*
}

Korean Ginseng Center and Ginseng Genetic Resource Bank, Kyung Hee University, Yongin 449-701, Korea

\begin{abstract}
The medicinal plant Panax ginseng (P. ginseng) contains various phytosterols and bioactive triterpene saponins (ginsenosides). Squalene synthase catalyzes the first committed step in ginsenoside biosynthesis. Transgenic plants of $P$. ginseng were generated by introducing the squalene synthase gene derived from $P$. ginseng. Adventitious roots of the transgenic ginseng grew best in B5 medium, and $2 \mathrm{~g}$ of inoculum secured an optimal growth rate. Two phytohormones, indolebutyric acid and 1-naphtalene acetic acid, increased root growth and decreased ginsenoside production. Treatment with two selected elicitors, chitosan and jasmonic acid, and a precursor of the isoprenoid pathway, mevalonic acid, enhanced ginsenoside production and retarded ginseng growth rate.
\end{abstract}

Keywords: Elicitor, Ginseng, Ginsenoside, Jasmonic acid, Squalene synthase

\section{INTRODUCTION}

Panax ginseng (P. ginseng) is one of the most wellknown oriental medicinal plants. Triterpene saponins (ginsenosides) extracted from the roots of $P$. ginseng have various kinds of biological activities [1]. Although more than 80 different skeletal types of triterpenes are known to occur naturally, the dammarane-type triterpenes are found only in a few species as major compounds, e.g., P. ginseng [2] and Gynostemma pentaphyllum (jiaogulan) [3]. Recent research including large-scale gene analysis [4-5] and proteome analysis [6-7] has shown that $P$. ginseng is one of the most suitable systems for studying the biosynthesis of dammarane-type triterpene saponins.

The isoprenoid biosynthetic pathway plays an important role in plant metabolism. The best-known metabolites in this pathway are sterols, gibberellins, carotenoids [8], and terpenes such as mono-, sesqui-, di-, and triterpenes
[9]. Overproduction of phytosterols and triterpenes by metabolic engineering may be an attractive strategy for producing pharmacologically active medicinal plants of superior quality. Squalene synthase (SQS) (EC 2.5.1.21) catalyzes the first enzymatic step in the central isoprenoid pathway toward sterol and triterpenoid biosynthesis [10]. As SQS acts at a putative key branching point in the isoprenoid biosynthetic pathway, it may have a regulatory role in this pathway. SQS has been investigated in animals [11], yeast [12], and plants [13-16].

Elicitor-induced accumulation of secondary metabolites has received much attention, and jasmonic acid has proven to be an effective elicitor [17-19]. Jasmonic acid is believed to be an integral component of signal transduction pathways resulting in the activation of defense responses and secondary metabolism [20,21]. It was reported that exogenously applied elicitors, particularly

\footnotetext{
(c) This is an Open Access article distributed under the terms of the Creative Commons Attribution Non-Commercial License (http://creativecommons.org/licenses/by-nc/3.0/) which permits unrestricted non-commercial use, distribution, and reproduction in any medium, provided the original work is properly cited.
}

Received 11 Jan. 2010, Revised 26 Feb. 2010, Accepted 11 Mar. 2010

*Corresponding author

E-mail: dcyang@khu.ac.kr

Tel: +82-31-201-2688, Fax: +82-31-201-2687 
jasmonic acid, stimulate the biosynthesis of many secondary metabolites when the appropriate type of elicitors are chosen in right concentration. In case of ginseng, jasmonic acid significantly improved ginsenoside production in the cultures of hairy roots [22-23]. Here, we report on the optimal media conditions for enhanced ginsenoside production by introducing the squalene synthase gene into ginseng. The effects of phytohormones and effective elicitors are also addressed.

\section{MATERIALS AND METHODS}

\section{Vector construction}

The entire coding region of the squalene synthase gene (PgSQS1) was amplified from P. ginseng cDNA, and the resulting 1248-b.p. PCR product was digested with $N c o$ I and BamHI and ligated into the same restriction sites between the double cauliflower mosaic virus $35 \mathrm{~S}$ promoter, followed by the alfalfa mosaic virus enhancer and the NOS terminator in pBI524 (NRC Plant Biotechnology Institute, Saskatoon, Canada). The pBI524-carrying PgSQS1 was digested with $\mathrm{XbaI}$, and the resulting fragment was ligated into the same restriction sites within pCAMBIA1302 (Cambia, Canberra, Australia). The final construct was transformed into ginseng using Agrobacterium tumefaciens LBA 4404.

\section{Genetic transformation of ginseng}

Genetic transformation of $P$. ginseng was carried out as described earlier [24]. Cotyledonary somatic embryos that survived on the selection medium containing $100 \mathrm{mg} / \mathrm{L}$ hygromycin and $200 \mathrm{mg} / \mathrm{L}$ cefotaxime were detached and transferred to MS medium [25] supplemented with $100 \mathrm{mg} / \mathrm{L} \mathrm{GA}_{3}$ and $100 \mathrm{mg} / \mathrm{L}$ hygromycin. The embryos were then germinated and maintained on half-strength MS medium with $2 \%$ sucrose. The culture room was maintained at $23^{\circ} \mathrm{C}$ under a $16 / 8$-h (light/ dark) cycle, with light supplied by white fluorescent tubes at an intensity of $24 \mu \mathrm{mol} / \mathrm{m}^{2} / \mathrm{s}^{2}[24]$.

\section{Adventitious root culture}

Adventitious roots $(0.5 \mathrm{~g})$ were grown in a $100-\mathrm{mL}$ Erlenmeyer flask with $40 \mathrm{~mL}$ of B5 [26] medium (pH 5.7) supplemented with indolebutyric acid (IBA) or 1-naphtalene acetic acid (NAA).The mother inoculum was subcultured every 30 days. The ginseng cell lines were grown in three media, namely MS $\left(20.6 \mathrm{mM} \mathrm{NH}_{4}^{+}\right.$ ion), B5 (2.0 $\mathrm{mM} \mathrm{NH}_{4}{ }^{+}$ion), and WPM [27], with 0.25 , $0.5,1$, and $2 \mathrm{~g} / \mathrm{L}$ inoculum (fresh weight). The grown roots were used for further studies. The culture room was maintained at $23^{\circ} \mathrm{C}$ under a $16 / 8$-h (light/dark) cycle, with light supplied by white fluorescent tubes at an intensity of $24 \mu \mathrm{mol} / \mathrm{m}^{2} / \mathrm{s}^{2}$ [24].

\section{Elicitor and mevalonic acid treatment}

Adventitious roots developed around the excised edges of the segments were detached from the maternal explants and subcultured in B5 medium with 3\% sucrose and $2 \mathrm{mg} / \mathrm{L}$ IBA. The culture room was maintained at $23^{\circ} \mathrm{C}$ under a $16 / 8$-h (light/dark) cycle, with light supplied by white fluorescent tubes at an intensity of $24 \mu \mathrm{mol} / \mathrm{m}^{2} / \mathrm{s}^{2}$ [24]. The elicitors, chitosan, jasmonic acid, and mevalonic acid, were added at different concentrations once the cultures reached the vigorous growth phase (after 4 weeks).

\section{Ginsenoside analyses by TLC and HPLC}

The ginsenosides were extracted twice using a mixture of water and n-butyl alcohol (1:1). The solvent was evaporated in vacuo. The residue redissolved in methanol was detected by thin layer chromatography (TLC) using a Silica gel plate 60 F-254 (Merck, Darmstadt, Germany). $\mathrm{CHCl}_{3}-\mathrm{MeOH}-\mathrm{H}_{2} \mathrm{O}$ (65:35:10, v/v, lower phase) was used as the developing solvent, and bands were detected by spraying $10 \% \mathrm{H}_{2} \mathrm{SO}_{4}$ on TLC plates, followed by heating at $110^{\circ} \mathrm{C}$. HPLC-grade acetonitrile and water were purchased from SK chemicals (Ulsan, Korea). The reaction mixture was extracted with $n$ butanol saturated with $\mathrm{H}_{2} \mathrm{O}$, which was evaporated in vacuo. The residue dissolved in $\mathrm{CH}_{3} \mathrm{OH}$ was applied to HPLC analysis. HPLC was performed with a C18 $(250 \times 4.6 \mathrm{mM}$, ID $5 \mu \mathrm{m})$ column, acetonitrile (solvent A), and distilled water (solvent B) at A:B ratios of 15:85, $21: 79,58: 42,90: 10,90: 10,15: 85$, and 15:85, with running times of 0 to 5,5 to 25,25 to 70,70 to 72,72 to 82 , 82 to 84 , and 84 to $100 \mathrm{~min}$, respectively, at a flow rate of $1.6 \mathrm{~mL} / \mathrm{min}$. The detection of ginsenoside was done at $203 \mathrm{~nm}[28]$.

\section{RESULTS AND DISCUSSION}

\section{Genetic transformation of $P_{\text {. ginseng }}$ and induc- tion of adventitious roots}

$P$. ginseng was transformed following the previously described method [24]. Hygromycin-resistant somatic embryos were developed on the surfaces of brown explants during the subculture on the selection medium. Selected cotyledonary embryos were germinated on MS medium supplemented with $100 \mathrm{mg} / \mathrm{L} \mathrm{GA}_{3}$ and $50 \mathrm{mg} /$ L hygromycin for 1 month. No morphological differ- 


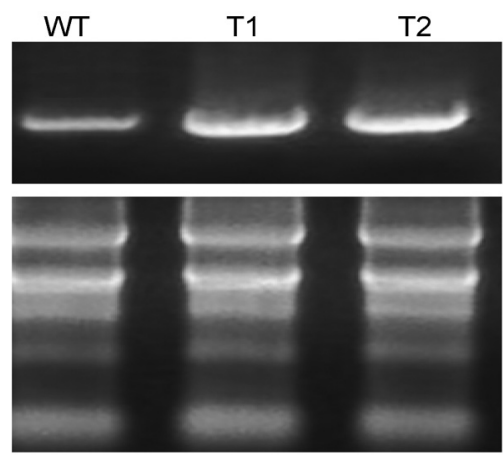

Fig. 1. RT-PCR results showing increased mRNA expression of squalene synthase gene (PgSQS1) in transgenic ginseng. Total RNA was used as the loading control (lower panel). The transcripts of PgSQS1 were greater in the transgenic lines (T1 and $\mathrm{T} 2$ ) than in non-transgenic wild-type ginseng (WT, upper panel).

ences were observed between transgenic and non-transgenic $P$. ginseng plants. The integration of $P g S Q S 1$ and hygromycin phosphotransferase (HPT) genes into the hygromycin-resistant plantlets was confirmed by PCR. The PCR product showed clear bands for the PgSQS1 (1,248 b.p.) and HPT genes (700 b.p.) (data not shown). RT-PCR analysis showed increased gene expression from the transgenic plants (Fig. 1).

Because ginseng grows extremely slowly in the field, roots cultured in vitro were used to analyze the characteristics of the transgenic plants. Leaves and stem segments of both transgenic and wild-type plants were cultured for 1 month to induce adventitious roots. The cultures were maintained by subculturing every 4 weeks. Adventitious roots derived from independent transgenic ginseng plants were regarded as independent lines.

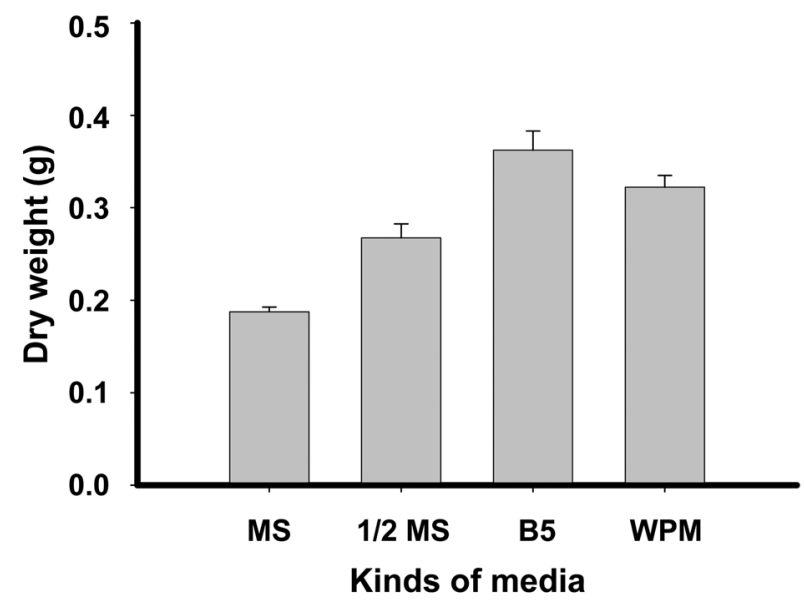

Fig. 2. Effect of different media on the growth of adventitious roots. Vertical bar indicates mean values \pm standard error $(n=5)$.

\section{Optimization of media conditions for ginsen- oside production}

Growth of transgenic adventitious roots reached a maximum in various media (0.36 g dry weight in B5 medium, $0.19 \mathrm{~g}$ dry weight in MS medium, $0.27 \mathrm{~g}$ dry weight in $1 / 2$ medium, and $0.32 \mathrm{~g}$ dry weight in WPM medium) by 30 days. The best result was observed on B5 medium (Fig. 2). The media differed in the concentration of $\mathrm{NH}_{4}^{+}$ions, which was $20.6 \mathrm{mM}$ in MS and 2.0 $\mathrm{mM}$ in $\mathrm{B} 5$. The high concentration of $\mathrm{NH}_{4}^{+}$ions in $\mathrm{MS}$ medium seemed to inhibit the growth of ginseng roots.

The size of the inoculum was also investigated, and the results are shown in Fig. 3. Net growth (total weight - inoculum weight) during the culture increased with increasing inoculum weight up to $2 \mathrm{~g}$.

\section{Effect of PgSQS1 overexpression on growth rate}

The growth rate of adventitious roots overexpressing PgSQS1 (PgSQS1ox) cultured in B5 liquid media supplemented with auxins was compared to that of the wild-type control (Fig. 4). Good proliferation of transgenic roots was observed in the media supplemented with IBA and NAA in the first 30 days. The dry weight of transgenic roots and wild type roots cultured with IBA and NAA increased rapidly until day 30 , after which the growth curves plateaued. The maximum growth of adventitious roots by day 30 varied with inoculum (transgenic adventitious roots, 0.27 and $0.24 \mathrm{~g}$ dry weight after treatment with $5 \mathrm{mg} / \mathrm{L}$ IBA and $5 \mathrm{mg} / \mathrm{L} \mathrm{NAA}$, respectively; non-transgenic adventitious roots, 0.3 and $0.34 \mathrm{~g}$ dry weight after treatment with $5 \mathrm{mg} / \mathrm{L}$ IBA and $5 \mathrm{mg} / \mathrm{L} \mathrm{NAA}$, respectively). The non-transgenic adventitious roots grew faster than the transgenic roots. The

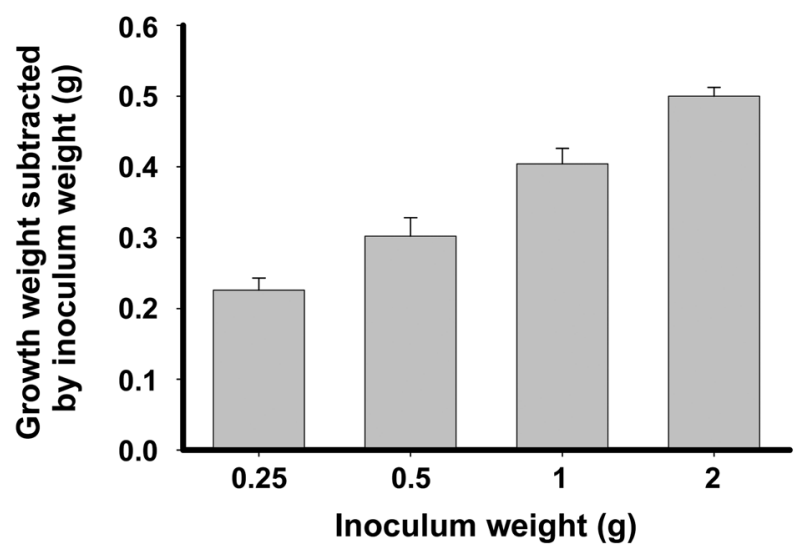

Fig. 3. Growth of adventitious transgenic ginseng root tips after 30 days of culture and for different amounts of inoculum. Vertical bars indicate mean values \pm standard error $(n=5)$. 
effects of NAA and IBA were similar. The ginsenoside levels of the transgenic adventitious roots were assayed by TLC (Fig. 5). Both transgenic and non-transgenic adventitious roots, as well as ginsengs in the field, showed similar levels of ginsenosides from $\mathrm{Rb} 1$ to $\mathrm{Rg} 1$. The adventitious roots of transgenic and non-transgenic plants assayed using TLC were subjected to HPLC. The levels of nine major ginsenoside compounds (Rb1, $\mathrm{Rb} 2$, Rc, Rd, Re, Rf, Rg1, Rg2, and Rg3) were determined by HPLC (Table 1). Ginsenosides Rb1, Rc, and Rd increased 2-, 3-, and 17-fold in the transgenic lines. The effect of auxins on ginsenoside production differed between transgenic and non-transgenic plants. In nontransgenic plants, auxin generally seemed to boost plant

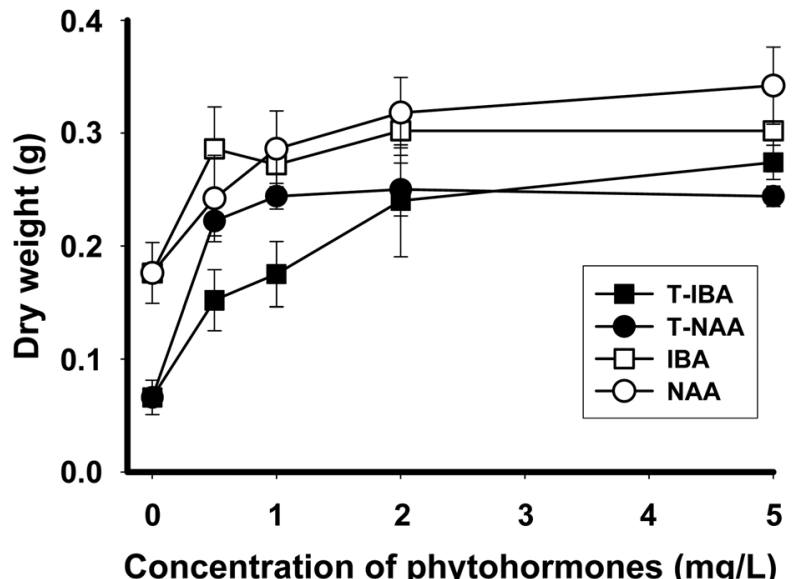

Fig. 4. In vitro growth of ginseng roots on media containing phytohormones. T-IBA, transgenic roots with indolebutyric acid (IBA); T-NAA, transgenic roots with naphtalene acetic acid (NAA); IBA, non-transgenic roots with IBA; NAA, non-transgenic roots with NAA. Vertical bars indicate mean values \pm standard error $(n=5)$. growth rate and inhibit total ginsenoside production. However, PgSQS1ox seemed to suppress the negative effects of auxin. This result led to the hypothesis that increased production of ginsenoside retards plant growth rate and results in decreased total dry weight, as shown in Fig. 4. These results suggest the possible interaction of phytohormones and ginsenoside production, which requires further experimental confirmation.

\section{Effective elicitors of ginsenoside production}

The growth rate of transgenic adventitious roots of $P$. ginseng and ginsenoside accumulation by overexpression of the PgSQS1 gene may be influenced by different elicitors and by the biosynthesis of mevalonate. The dry weight of roots of untreated transgenic cultures averaged $0.27 \mathrm{~g}$ per flask (Fig. 6), indicating that the tested elicitors drastically affected growth rate, resulting in decreased dry weight. But no significant effect of mevalonic acid was observed (Fig. 6). Total production

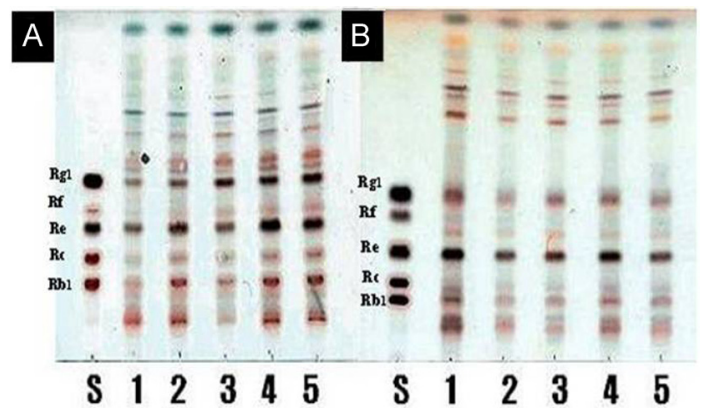

Fig. 5. Thin layer chromatography patterns of ginsenoside in several lines of Panax ginseng. (A) transgenic adventitious roots, (B) non-transgenic adventitious roots. S, standard.

Table 1. Production of ginsenosides in adventitious roots of transgenic and non-transgenic Panax ginseng

\begin{tabular}{|c|c|c|c|c|c|c|c|c|c|c|c|c|}
\hline \multirow{2}{*}{ Cell line } & \multirow{2}{*}{ Treatment } & \multirow{2}{*}{$(\mathrm{mg} / \mathrm{L})$} & \multicolumn{10}{|c|}{ Ginsenoside content (mg/g dry weight) } \\
\hline & & & $\mathrm{Rb} 1$ & $\mathrm{Rb} 2$ & $\mathrm{Rc}$ & $\mathrm{Rd}$ & $\operatorname{Rg} 1$ & $\operatorname{Rg} 2$ & $\operatorname{Rg} 3$ & $\mathrm{Rf}$ & $\operatorname{Re}$ & Total \\
\hline \multirow{5}{*}{$\begin{array}{c}\text { Non- } \\
\text { transgenic }\end{array}$} & Non-treatment & 0.0 & 0.45 & 0.19 & 0.07 & 0.01 & 0.20 & 0.19 & 0.00 & 0.02 & 1.25 & 2.36 \\
\hline & \multirow{2}{*}{ NAA } & 1.0 & 0.29 & 0.32 & 0.03 & 0.03 & 0.12 & 0.11 & 0.00 & 0.01 & 0.88 & 1.76 \\
\hline & & 5.0 & 0.23 & 0.15 & 0.02 & 0.01 & 0.10 & 0.03 & 0.00 & 0.00 & 0.62 & 1.15 \\
\hline & \multirow{2}{*}{ IBA } & 1.0 & 0.46 & 0.36 & 0.04 & 0.03 & 0.18 & 0.13 & 0.00 & 0.03 & 1.41 & 2.62 \\
\hline & & 5.0 & 0.26 & 0.28 & 0.03 & 0.01 & 0.16 & 0.13 & 0.00 & 0.01 & 0.90 & 1.75 \\
\hline \multirow{5}{*}{ Transgenic } & Non-treatment & 0.0 & 0.96 & 0.24 & 0.23 & 0.17 & 0.16 & 0.12 & 0.00 & 0.03 & 1.24 & 3.13 \\
\hline & \multirow{2}{*}{ NAA } & 1.0 & 1.78 & 0.47 & 0.47 & 0.31 & 0.27 & 0.16 & 0.00 & 0.03 & 1.69 & 5.17 \\
\hline & & 5.0 & 2.10 & 0.41 & 0.33 & 0.53 & 0.67 & 0.17 & 0.03 & 0.04 & 1.69 & 5.94 \\
\hline & \multirow{2}{*}{ IBA } & 1.0 & 2.28 & 0.62 & 0.49 & 0.59 & 0.57 & 0.36 & 0.01 & 0.05 & 2.69 & 7.65 \\
\hline & & 5.0 & 2.51 & 0.68 & 0.44 & 0.58 & 0.67 & 0.10 & 0.01 & 0.01 & 1.77 & 6.75 \\
\hline
\end{tabular}

Each value is presented as mean value of two experiments $(p<0.003)$.

NAA, naphtalene acetic acid; IBA, indolebutyric acid. 
Table 2. Overproduction of ginsenosides by elicitors and a precursor in the transgenic roots

\begin{tabular}{|c|c|c|c|c|c|c|c|c|c|c|}
\hline \multirow{2}{*}{ Elicitor } & \multicolumn{10}{|c|}{ Ginsenoside (mg/g dry weight) } \\
\hline & $\mathrm{Rb} 1$ & $\mathrm{Rb} 2$ & $\mathrm{Rc}$ & $\mathrm{Rd}$ & $\operatorname{Rg} 1$ & $\operatorname{Rg} 2$ & $\operatorname{Rg} 3$ & $\mathrm{Rf}$ & $\operatorname{Re}$ & Total \\
\hline Non-transgenic & 1.00 & 0.47 & 0.29 & 0.15 & 0.54 & 0.46 & 0.00 & 0.06 & 4.08 & 7.04 \\
\hline Transgenic & 6.77 & 1.29 & 1.07 & 1.08 & 1.05 & 0.54 & 0.01 & 0.08 & 5.68 & 17.57 \\
\hline Chitosan $(50 \mathrm{mg} / \mathrm{L})$ & 10.10 & 2.03 & 1.27 & 0.83 & 0.69 & 0.43 & 0.03 & 0.09 & 3.47 & 18.92 \\
\hline Jasmonic acid $(10 \mathrm{mg} / \mathrm{L})$ & 32.74 & 13.27 & 11.21 & 9.81 & 1.28 & 2.02 & 0.24 & 0.43 & 13.48 & 84.46 \\
\hline Mevalonic acid $(50 \mathrm{mg} / \mathrm{L})$ & 9.52 & 2.53 & 1.47 & 1.55 & 2.26 & 1.04 & 0.03 & 0.19 & 8.93 & 27.51 \\
\hline
\end{tabular}

Each value is presented as mean value of two experiments $(p<0.003)$.

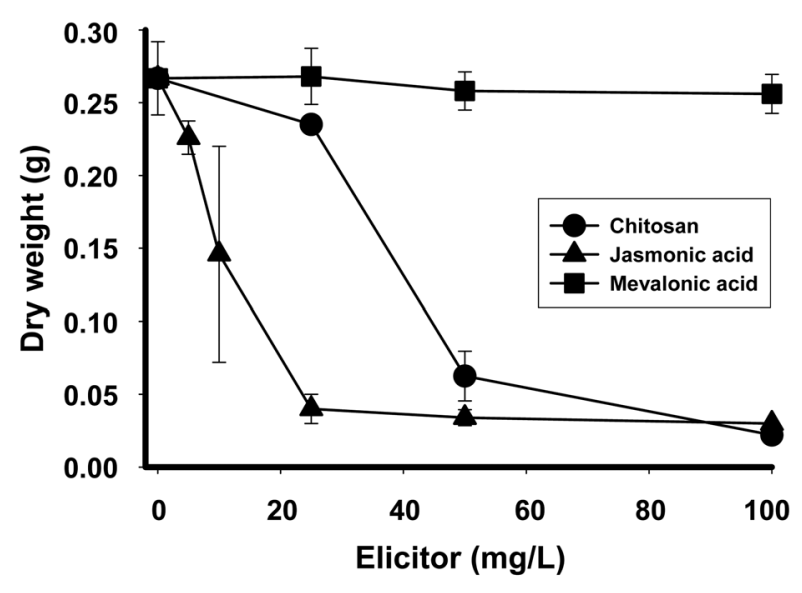

Fig. 6. Effects of elicitors and the precursor (mevalonic acid) on the growth of transgenic adventitious roots. Roots were cultured in B5 medium ( $\mathrm{pH}$ 5.7) supplemented with $2 \mathrm{mg} / \mathrm{L} \mathrm{IBA}$ in 100-mL flasks, each containing $40 \mathrm{~mL}$ of the medium, on a rotary shaker shaking $100 \mathrm{rpm}$ at $0.5 \mathrm{~g}$ and subcultured every 30 days. Vertical bars indicate mean values \pm standard error $(n=5)$.

of ginsenosides increased markedly with the addition of elicitors and the precursor (Table 2). Ginsenoside analysis by HPLC revealed that the total ginsenoside content in the transgenic $P$. ginseng roots after 30 days of culture was maximized ( $84.46 \mathrm{mg} / \mathrm{g}$ dry weight) by the addition of jasmonic acid; this level was 12 times higher than that of the wild type (Table 2). In conclusion, overexpression of PgSQS1 resulted in increased production of ginsenosides, as reported previously [16]. The optimal growth medium for increased ginsenoside production was found to be a B5 medium. Interestingly, the amount of ginsenosides in a ginseng plant seems to be negatively modulated by phytohormones and positively modulated by elicitors, which results in increased growth rates from phytohormoes and reduced growth rates from elicitors. Therefore, the effects of the physiological interaction of growth-regulating hormones and growth-retarding elicitors on ginsenoside levels will require future study.

\section{ACKNOWLEDGEMENTS}

This study was supported by Korean Ginseng Center for Most Valuable Products \& Ginseng for Technology Development Program of Agriculture and Forestry, Ministry of Food, Agriculture, Forestry and Fisheries, Republic of Korea.

\section{REFERENCES}

1. Briskin DP. Medicinal plants and phytomedicines. Linking plant biochemistry and physiology to human health. Plant Physiol 2000;124:507-514.

2. Kushiro T, Ohno Y, Shibuya M, Ebizuka Y. In vitro conversion of 2,3-oxidosqualene into dammarenediol by Panax ginseng microsomes. Biol Pharm Bull 1997;20:292-294.

3. Cui J, Eneroth P, Bruhn JG. Gynostemma pentaphyllum: identification of major sapogenins and differentiation from Panax species. Eur J Pharm Sci 1999;8:187-191.

4. Jung JD, Park HW, Hahn Y, Hur CG, In DS, Chung HJ, Liu JR, Choi DW. Discovery of genes for ginsenoside biosynthesis by analysis of ginseng expressed sequence tags. Plant Cell Rep 2003;22:224-230.

5. Kim MK, Lee BS, In JG, Sun H, Yoon JH, Yang DC. Comparative analysis of expressed sequence tags (ESTs) of ginseng leaf. Plant Cell Rep 2006;25:599-606.

6. Kim SI, Kim JY, Kim EA, Kwon KH, Kim KW, Cho K, Lee JH, Nam MH, Yang DC, Yoo JS, et al. Proteome analysis of hairy root from Panax ginseng C.A. Meyer using peptide fingerprinting, internal sequencing and expressed sequence tag data. Proteomics 2003;3:2379-2392.

7. Nam MH, Heo EJ, Kim JY, Kim SI, Kwon KH, Seo JB, Kwon O, Yoo JS, Park YM. Proteome analysis of the responses of Panax ginseng C. A. Meyer leaves to high light: use of electrospray ionization quadrupole-time of flight mass spectrometry and expressed sequence tag data. Proteomics 2003;3:2351-2367.

8. Bloch K. Sterol molecule: structure, biosynthesis, and function. Steroids 1992;57:378-383. 
9. Chappell J. The Biochemistry and Molecular Biology of Isoprenoid Metabolism. Plant Physiol 1995;107:1-6.

10. Abe I, Rohmer M, Prestwich GD. Enzymatic cyclization of squalene and oxidosqualene to sterols and triterpenes. Chem Rev 1993;93:2189-2206.

11. Guan G, Dai P, Shechter I. Differential transcriptional regulation of the human squalene synthase gene by sterol regulatory element-binding proteins (SREBP) 1a and 2 and involvement of 5' DNA sequence elements in the regulation. J Biol Chem 1998;273:12526-12535.

12. Kennedy MA, Bard M. Positive and negative regulation of squalene synthase (ERG9), an ergosterol biosynthetic gene, in Saccharomyces cerevisiae. Biochim Biophys Acta 2001;1517:177-189.

13. Vogeli U, Chappell J. Induction of sesquiterpene cyclase and suppression of squalene synthetase activities in plant cell cultures treated with fungal elicitor. Plant Physiol 1988;88:1291-1296.

14. Devarenne TP, Ghosh A, Chappell J. Regulation of squalene synthase, a key enzyme of sterol biosynthesis, in tobacco. Plant Physiol 2002;129:1095-1106.

15. Wentzinger LF, Bach TJ, Hartmann MA. Inhibition of squalene synthase and squalene epoxidase in tobacco cells triggers an up-regulation of 3-hydroxy-3-methylglutaryl coenzyme a reductase. Plant Physiol 2002;130:334-346.

16. Lee MH, Jeong JH, Seo JW, Shin CG, Kim YS, In JG, Yang DC, Yi JS, Choi YE. Enhanced triterpene and phytosterol biosynthesis in Panax ginseng overexpressing squalene synthase gene. Plant Cell Physiol 2004;45:976984.

17. Aerts RJ, Gisi D, Carolis ED, Luca VD, Baumann TW. Methyl jasmonate vapor increase the developmentally controlled synthesis of alkaloids in Cartharanthus and Cinchona seedlings. Plant J 1994;5:635-643.

18. Gundlach H, Muller MJ, Kutchan TM, Zenk MH. Jasmonic acid is a signal transducer in elicitor-induced plant cell cultures. Proc Natl Acad Sci USA 1992;89:2389-2393.

19. Ketchum RE, Gibson DM, Croteau RB, Shuler ML. The kinetics of taxoid accumulation in cell suspension cultures of Taxus following elicitation with methyl jasmonate. Biotechnol Bioeng 1999;62:97-105.

20. Farmer EE, Caldelari D, Pearce G, Walker-Simmons MK, Ryan CA. Diethyldithiocarbamic acid inhibits the octadecanoid signaling pathway for the wound induction of proteinase inhibitors in tomato leaves. Plant Physiol 1994; 106:337-342.

21. Doares SH, Syrovets T, Weiler EW, Ryan CA. Oligogalacturonides and chitosan activate plant defensive genes through the octadecanoid pathway. Proc Natl Acad Sci USA 1995;92:4095-4098.

22. Yu KW, Gao W, Hahn EJ, Paek KY. Jasmonic acid improves ginsenoside accumulation in adventitious root culture of Panax ginseng C.A. Meyer. Biochem Eng J 2002;11:211-215.

23. Yu KW, Gao WY, Son SH, Paek KY. Improvement of ginsenoside production by jasmonic acid and some other elicitors in hairy root culture of ginseng (Panax ginseng $\mathrm{C}$. A. Meyer). In Vitro Cell Dev Biol Plant 2000;36:424-428.

24. Choi YE, Yang DC, Kusano T, Sano H. Rapid and efficient Agrobacterium-mediated transformation of Panax ginseng by plasmolyzing pre-treatment of cotyledons. Plant Cell Rep 2001;20:616-621.

25. Murashige T, Skoog F. A revised medium for rapid growth and bioassays with tobacco tissue cultures. Physiol Plant 1962;15:473-497.

26. Gamborg OL, Miller RA, Ojima K. Nutrient requirements of suspension cultures of soybean root cells. Exp Cell Res 1968;50:151-158.

27. Lloyd G, McCown B. Commercially-feasible micropropagation of mountain laurel, Kalmia latifolia, by use of shoot-tip culture. Comb Proc Int Plant Prop Soc 1980;30:421-427.

28. Cheng LQ, Na JR, Bang MH, Kim MK, Yang DC. Conversion of major ginsenoside Rb1 to 20(S)-ginsenoside Rg3 by Microbacterium sp. GS514. Phytochemistry 2008;69:218-224. 